\title{
Der 14. Kinder- und Jugendbericht
}

\section{Ein Jahr danach}

Anfang 2013 wurde der 14. Kinder- und Jugendbericht der Bundesregierung veröffentlicht. Seitdem findet eine umfängliche Befassung mit dem Bericht in kinder- und jugendpolitischen Kontexten, in der Trägerlandschaft sowie in der Wissenschaft statt. Viele Inhalte des thematisch breit aufgestellten Gesamtberichts werden darin aufgenommen, kritisch diskutiert oder auch darin aufgeworfene Perspektiven als Orientierung für die Gestaltung von politischen wie praxisbezogenen Entwicklungen genutzt.

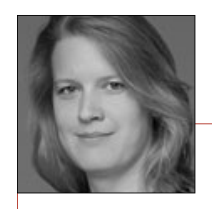

Nadia Kutscher *1972

Dr. Professorin für Soziale Arbeit und Ethik an der Universität Vechta, Mitglied der Sachverständigenkommission für den 14. Kinder- und Jugendbericht der Bundesregierung. nadia.kutscher@ uni-vechta.de

lich einladen.

Dieser Schwerpunkt möchte nach einem Jahr der Auseinandersetzung mit dem Bericht ein Forum eröffnen, das einen Blick auf die bisherige Diskussion, ein „Zwischenfazit“ ermöglicht und auch Perspektiven für die weitere Befassung mit den darin angesprochenen Themen aufmacht. Diese diskursive Auseinandersetzung mit dem Bericht wird darüber hinaus in einem gemeinsamen Fachforum der Sozial Extra und des Erziehungswissenschaftlichen Fakultätentags „Ein Jahr danach - Der 14. Kinder- und Jugendbericht der Bundesregierung in der Diskussion" am 4. Juni 2014 auf dem 15. Kinder- und Jugendhilfetag in Berlin fortgesetzt, zu dem wir herz-

Insbesondere die in Teil D (Kapitel 1-15) dargestellten Herausforderungen für die Kinder- und Jugendhilfe beinhalten viele Anregungen und Konkretisierungen von Konsequenzen, die sich aus den Analysen des Berichts ergeben und wollen dabei eine Grundlage für eine zukunftsgerichtete Befassung mit aktuellen Themen der Kinder- und Jugendhilfe anbieten. Bislang kann festgestellt werden, dass sich diese Auseinandersetzung auf den verschiedenen Ebenen durch ein hohes Interesse an den Inhalten und Markierungen des Berichts auszeichnet, durch Kontroversen an verschiedenen Stellen, interessengeleitete Positionierungen verschiedener AkteurInnen sowie die interessierte Befassung mit neu aufgeworfenen Perspektiven, wie z.B. den neuen Mischungsverhältnissen von öffentlicher und privater Verantwortung, der Ungleichheitsreproduktion im Kontext der Kinder- und Jugendhilfe, der Frage nach der finanziellen Leistungsfähigkeit und einer entsprechenden Entlastung der Kommunen sowie der Mediatisierung der Kinder- und Jugendhilfe. Während in politischen Kontexten sich Folgen und Entwicklungen vor dem Hintergrund der im Bericht aufgeworfenen Fragen und Handlungsoptionen bislang teils noch nicht unbedingt deutlich abzeichnen, ist im Kontext der Fachszene in vielerlei Hinsicht einiges an Bewegung zu beobachten. So befassen sich mehr und mehr Träger mit der Frage der Mediatisierung des Aufwachsens von Kindern und Jugendlichen und auch den Implikationen, die eine zunehmend mediatisierte und technisierte Kinder- und Jugendhilfe mit sich bringt. Die Frage nach der eigenen - impliziten wie expliziten - Beteiligung von Kinder- und Jugendhilfe an der Reproduktion von Ungleichheit durch Angebotsformen, Handeln, aber auch durch politische Steuerung stößt auf viel Resonanz. Die Frage nach einer Jugendpolitik, die Kinder nicht außer acht lässt, Jugendliche aber als AkteurInnen in den Blick nimmt, die nicht nur unter Produktivitäts- bzw. Employabilitygesichtspunkten oder Risikoverdacht wahrgenommen werden, beschäftigt Politik wie Trägerorganisationen. Die Auseinandersetzung mit der Bedeutung neuer Verantwortungsverhältnisse im Kontext von Produktivitätsdispositiven, Kontrolltendenzen, Unterstützungsbedarfen und Ungleichheitsbearbeitung bedarf einer längerfristigen, multidimensionalen Reflexion, die auf die Weiterentwicklung praktischer Angebotsstrukturen, konzeptioneller Ansätze und politischer Steuerungsmodi weitreichende Auswirkungen haben kann.

In diesem Schwerpunkt positionieren sich KollegInnen aus Trägerverbänden, Hochschule und Politik zu den bisherigen Auseinandersetzungen mit dem Kinder- und Jugendbericht. Karlheinz Struzyna blickt auf die politische Befassung mit Berichten insgesamt und dem 14. Kinder- und Jugendbericht im Besonderen. Ruth Enggruber diskutiert das im Bericht u.a. zugrunde gelegte „Wohlfahrtsviereck“ am Beispiel des Handlungsfeldes Übergang Schule-Beruf. Heinz-Josef Kessmann fokussiert verschiedene zentrale Thesen aus dem Bericht und kommentiert sie aus Trägerperspektive.

Abstract / Das Wichtigste in Kürze Der 14. Kinder- und Jugendbericht ist seit einem Jahr in der öffentlichen Diskussion. Zeit, dieser Debatte auch in Sozial Extra ein Forum zu geben.

Keywords/Stichworte $\quad$ 14. Kinder- und Jugendbericht, öffentliche und private Verantwortung, Kinder- und Jugendhilfe 